\title{
LOS HITOS CRÍTICOS DE LA TRAYECTORIA LABORAL ASCENDENTE DE PERUANAS Y PERUANOS EN CHILE ${ }^{1}$
}

\section{The critical milestones of the upward work path of peruvian men and women in Chile}

\author{
Camila León $\mathrm{Gin}^{2}$ \\ Universidad Alberto Hurtado, Santiago, Chile.
}

Recibido: 11 de marzo de 2011. Aprobado: 2 de junio de 2011.

\section{RESUMEN}

El siguiente artículo examina la trayectoria de peruanos y peruanas en el mercado del trabajo chileno de acuerdo a los hitos críticos o puntos de inflexión en su recorrido, para luego explorar sus efectos en el trayecto laboral ascendente de estos inmigrantes. Se indaga en los discursos, percepciones y significaciones que surgen de la experiencia en el trabajo en Chile a través de la realización de 32 relatos de vida a hombres y mujeres con una trayectoria laboral ascendente en Chile. Este trabajo da cuenta, mediante el análisis de los discursos, de que los principales hitos que determinan la trayectoria laboral de inmigrantes peruanos en Chile son los hitos laborales, histórico-sociales, familiares y personales, los cuales inciden en su ascenso laboral en la medida en que se hace uso de las redes y el capital simbólico con el que cuentan. Además se expresa la relevancia del contexto y sus decisiones personales a la hora de conjugar su situación particular con la estructura social en Chile.

PALABRAS CLAVE: Hitos críticos, migración, género.

\begin{abstract}
The following article examines the employment experiences of Peruvian men and women in the Chilean labor market, looking at critical milestones in order to explore the effects

$1 \quad$ Este artículo forma parte del proyecto Fondecyt № 1100793 titulado "Trayectorias laborales de los inmigrantes peruanos en Chile: estrategias de estructuración de la movilidad ocupacional ascendente”.

2 Bachiller en Ciencias Sociales, licenciada en Sociología y socióloga por la Universidad Alberto Hurtado. Correo electrónico: cami.leongin@gmail.com
\end{abstract}


of these breakthroughs on the advancing employment history of these immigrants. Discourses, perceptions, and meanings that arise from their experiences working in Chile are explored through the life stories of 32 Peruvian men and women who have improved their labor situations in Chile. By analyzing these discourses, this study finds that the primary milestones that determine the labor experience of Peruvian immigrants in Chile are related to work achievements, the socio-historical context, and family and personal aspects (improving their position by means of social networks and symbolic capital). Their living situation and personal decisions in relation to the Chilean social structure are also found to be relevant.

KEYWORDS: Critical milestones, migration, gender.

\section{INTRODUCCIÓN}

Analizar el recorrido de hombres y mujeres en el mercado del trabajo es relevante y sociológicamente pertinente, pues está determinado por estructuras sociales arraigadas (estratificación social) y también por procesos personales, sociales e históricos (Bourdieu 1997, 1979; Dávolos 2001; Pries 1997; Jiménez 2009) que culminan en puntos de inflexión de las trayectorias laborales. En este caso particular, es interesante para la sociología indagar sobre los fenómenos sociales como la migración laboral peruana, examinar estructuras como el funcionamiento del mercado del trabajo chileno e inquirir en las significaciones que las personas asignan a dichos procesos.

La trayectoria laboral es la orientación que toma la vida de las personas en el campo laboral. Esta se define de manera no lineal a través del tiempo, de acuerdo a las experiencias, acciones y prácticas (Araujo, Guzmán y Mauro 2000) que resultan de la mediación entre los condicionamientos externos y los individuales. El primer condicionamiento (plano externo) se refiere al mercado laboral chileno y a la segregación laboral de género y hacia el migrante, que se constituyen como problemas de inserción en el trabajo inicial en Chile y luego como obstáculos para la ascendencia. El segundo condicionamiento (plano interno o individual) son los recursos con los que cuentan ya sean sus conocimientos o calificaciones previas a la llegada, como también su capital económico, social y/o cultural- y las percepciones personales respecto de sus itinerarios en el mercado del trabajo. Se indagará en ambos condicionamientos, aunque se profundizará en este último. 
Las trayectorias laborales se componen por un conjunto de hitos críticos que se ven ampliamente influidos por situaciones de las condiciones externas y/o individuales, los cuales son "coyunturas específicas que exigen de los trabajadores decisiones y/o nuevas respuestas que ponen en juego sus motivaciones, sus recursos y representaciones e influyen sobre la dirección posterior que toma el itinerario laboral" (Guzmán, Arujo y Mauro 1999:145). Estos puntos de inflexión reflejan momentos históricos de la sociedad en general y también capturan decisiones y etapas de las personas en particular.

Se examinará, de acuerdo a la propia percepción de los inmigrantes, cuáles son los hitos que ellos consideran marcan un punto de inflexión e influyen en su trayectoria laboral en Chile. Luego se indagará la medida en que dichos hitos determinan la ascendencia de las trayectorias; ello, a través del análisis de los discursos y percepciones que los protagonistas tienen de sus recorridos. Finalmente se explorarán las trayectorias y sus hitos críticos, relevando semejanzas y diferencias de los discursos entre hombres y mujeres.

\section{INSERCIÓN LABORAL DE MIGRANTES}

Las trayectorias laborales son el resultado de la mediación entre los condicionamientos externos e internos. En el plano externo, un condicionante es la migración de peruanos a Chile, la cual está principalmente motivada por la integración laboral y económica, ya que el trabajo les permite ser valorados en la sociedad (Migueléz 2002) y se constituye como una de las esferas principales para elevar las condiciones de vida de los sujetos. Por lo tanto, la migración peruana a Chile es un fenómeno esencialmente económico-laboral (Stefoni 2009; Texidó et al. 2003; Migueléz 2002; Giorguli 2009).

El mercado laboral chileno funciona de manera diferente y desigual entre hombres y mujeres, lo que se sustenta en una sobrerrepresentación de las últimas en empleos que reproducen su rol tradicional y su situación de subordinación, lo que también incide en su trayectoria laboral. La tasa de participación femenina en el país ha experimentado un incremento en la última década, pasando de un 34,6 por ciento en 1996 a un 38,5 por ciento en 2006 (Díaz y Mella 2007:1). En el año 2009, las mujeres lograron un récord de participación en el mercado laboral. De acuerdo al Instituto Nacional de Estadística (INE), el 41,3 por ciento de las mujeres en edad de trabajar formó parte de la fuerza laboral. En cuanto a la educación, la mujer tiene mayor posibilidad de participación laboral al aumentar sus años de escolaridad, lo que no implica necesariamente ventaja en las 
remuneraciones, como sí sucede con los hombres (Servicio Nacional de la Mujer 2002, 2003; Valdés y Gomariz 1994).

Considerado el total nacional según rama de actividad, casi tres cuartas partes (44 por ciento) del empleo femenino (Díaz y Mella 2007:2) se concentran en los sectores de servicios sociales y personales, y en el comercio. La mujeres se ubican en un conjunto reducido de empleos que son definidos socialmente como femeninos pero además están ausentes en los directorios de organizaciones e instituciones, desenvolviéndose en cargos de menor jerarquía, prestigio y poder de decisión. En el caso de los hombres, la concentración según ramas de actividad se establece mayoritariamente en la construcción, industria manufacturera, explotación de minas y canteras, y servicios. Respecto de los tramos de remuneraciones, las brechas más significativas se consignan en los tramos más altos en el caso de los hombres y en los tramos más bajos para las mujeres (Sernam 2002, 2003; Díaz y Mella 2007, Henriquez 2006; Valdés y Gomariz 1994). Tal como se puede apreciar, el mercado de trabajo chileno en el cual se incorporan los inmigrantes peruanos/as está segregado, diferenciado y contiene brechas de género importantes. Ahora bien, es necesario estimar la población inmigrante que se integra en el mercado laboral de dichas características.

Texidó et al. (2003) señalan que en 2002 residían en Chile 220.000 extranjeros, los que representaban aproximadamente el 1,3 por ciento de la población total (según Censo 2002). La mayoría de los inmigrantes provenía de Argentina, seguida de Perú, Bolivia y Ecuador. Sin embargo, la información actual del Departamento de Extranjería del Ministerio del Interior permite identificar una nueva tendencia: "la migración peruana superó por primera vez a la argentina" (Stefoni 2009:4). Lo nuevo de la migración peruana, por lo tanto, no es la migración fronteriza, sino el incremento y la llegada de personas que provienen de diversos lugares de Perú y que llegan a Santiago (Stefoni 2009). Esta migración se caracterizó desde temprano por la alta presencia femenina (índice de masculinidad ${ }^{3}$ de 0,66 en el Censo 2002), situación que se mantuvo similiar en la Encuesta de caracterización socioeconómica (Casen) 2006, y la concentracion etaria en los tramos 15-29 y 30-34. ${ }^{4}$ Se trata de una migración en edad laboral, con alta presencia de mujeres (Stefoni 2009).

Otro condicionante del plano externo es la segregación laboral hacia el migrante y de género. Un análisis de las ocupaciones de los solicitantes de visa provenientes de los países vecinos, señala que alrededor del 85 por ciento de los varones se desempeñan como: obreros y jornaleros, trabajadores de servicios personales, y empleados de oficinas

3 La proporción de hombres frente a la de mujeres en la población.

4 La población peruana que llega a la capital mayoritariamente tiene entre 15 y 34 años en edad laboralmente activa. 
y afines. En el caso de las mujeres, el 90 por ciento se concentra en ocupaciones como: trabajadores de servicios personales y empleadas de oficinas y afines (Texidó et al. 2003). La fuerza de trabajo migrante se concentra principalmente en las ramas de servicios y comercio (ambos sectores absorben a más de la mitad de la Población económicamente activa "PEA" migrante); le siguen en importancia la industria, las finanzas, el transporte, la agricultura y, por último, la construcción (Texidó et al. 2003; Cortés 2005). Esta segregación ocupacional a la que se enfrentan los inmigrantes peruanos se constituye como obstáculo para la movilidad -por ende la ascendencia-, pues limita la posibilidad de inserción en otros espacios, rubros, puestos y tipos de trabajo.

En el mercado laboral chileno, este tipo de inserción de peruanos al trabajo es frecuente; sin embargo, es posible distinguir otro grupo minoritario de profesionales, técnicos y peruanos altamente calificados que tienen distintas trayectorias y procesos de integración al mercado del trabajo y que se insertan en sectores medios con mayores oportunidades de movilidad y ascendencia (Stefoni 2009; Henríquez y Riquelme 2006). Cabe destacar que dicha insersión, que profundizaremos luego, está determinada por su capital simbólico ${ }^{5}$ la ocupación en la que se desenvuelven en Chile.

Respecto de los condicionamientos del plano interno, los recursos económicos y sociales, sumado a los recursos simbólicos y culturales con los que viene este grupo minoritario de peruanos, se constituyen como ventajas elementales a la hora de construir su itinerario laboral en Chile. Las ventajas que tienen las personas con un alto capital cultural (Bourdieu y Passeron 1977) y social (Coleman 1998), es que alcanzan mayores y mejores logros educativos. Estos atributos son transmitidos, a su vez, por las familias de origen y reforzados a través de la interacción con amigos y otros miembros que participan del mismo contexto social y cultural, lo que finalmente se traduce en la obtención de mejor información y contactos al momento de insertarse en el mercado del trabajo (León y Martínez 2001). Lo anterior explica la relevancia del capital cultural en la inserción en el mercado laboral (Torche y Wormald 2004) para chilenos y peruanos. Sin embargo, en el caso de estos últimos, dada la discriminación y segregación, ellos requieren mayores credenciales que habiliten sus capacidades en el trabajo y, por ende, requieren del uso de otro tipo de capitales para insertarse laboral y económicamente.

5 Capital simbólico es un concepto acuñado por Pierre Bourdieu, quien argumenta que el capital económico considera el conjunto de recursos materiales a disposición de los agentes (dinero), el capital cultural es el capital informacional (lenguaje, conocimiento y certificaciones académicas) y finalmente el capital social es la suma de los recursos individuales o de grupo en lo referido a una red duradera de relaciones y de reconocimiento. Los tres tipos de capital requieren de capital simbólico para ser percibidos y reconocidos como legítimos socialmente (Bourdieu 1995). 
Aun cuando la inserción laboral de inmigrantes es heterogénea internamente, el presente estudio se enfocará en trayectorias laborales ascendentes. Entenderemos la trayectoria tomando en cuenta el primer trabajo desempeñado en Perú -como punto de referencia para comparación- hasta el trabajo actual en Chile. La ascendencia será analizada desde dos esferas: el plano subjetivo, es decir, la forma como perciben los inmigrantes su ascenso laboral, y el plano objetivo: mejorías en condiciones económicas, sociales o personales tales como aumento del ingreso, condiciones de trabajo, estatus social, contratación, acceso a beneficios sociales, entre otros.

Se desprende de lo anterior la pertinencia de un análisis de movilidad social producto de la trayectoria laboral ascendente de los inmigrantes. Es notoria la existencia de una estrecha relación entre los desplazamientos que efectúan los individuos, familias o grupos dentro de un determinado grupo socioeconómico y al interior del mercado laboral. Sin embargo, la siguiente investigación pretende indagar sobre los trayectos e hitos que los marcan; no busca analizar el impacto en su posición social, sino se posiciona desde la sociología del trabajo y el fenómeno de la migración para indagar los elementos que producen el paso de un trabajo a otro mejor que el anterior (en momentos específicos de su recorrido laboral).

Por otra parte, para realizar un análisis de movilidad social sería esencial tomar en cuenta la estratificación social de ambos países, la estructura de clases, las posiciones socioeconómicas de los entrevistados y cómo estas fluctúan en su vida (Bourdieu 1984; Crompton 1994; Erikson y Goldthorpe 1993; Goldthorpe 1987; Ram 2008; Wright 1992). Lo anterior funciona de manera diferenciada para chilenos y peruanos, y entre los peruanos en su país de origen y los peruanos que migraron a Chile, por ende, la movilidad horizontal (el paso de los individuos, grupo, rama o círculo ideológico a otro, sin que esto implique la alteración del estatus social) o vertical (se refiere al cambio de una clase a otra) no podrían ser medidos con los mismos indicadores -tales como la educación y trabajo- de los chilenos, pues, como hemos argumentado, los inmigrantes peruanos requieren legitimarse con mayores y distintas credenciales que los nacionales.

La pregunta de investigación que guiará el artículo es: ¿cuáles son los hitos críticos que condicionan la trayectoria laboral ascendente de inmigrantes peruanos en Chile? Se intentará examinar cuáles son los hitos críticos de la trayectoria laboral de peruanos y peruanas en Chile. Además se explorará la incidencia de los hitos en el ascenso del recorrido laboral de inmigrantes. Finalmente se pretende analizar las similitudes y diferencias entre los hitos críticos que determinan la ascendencia de peruanos y peruanas. 


\section{Metodología}

En este estudio el abordaje de las trayectorias laborales se hizo utilizando una estrategia de investigación de carácter cualitativo, vale decir, se centra en los discursos y significados en torno a las trayectorias laborales, usando relatos de vida laboral. En este caso, focalizado en la experiencia laboral en el marco migratorio. Esta técnica permite que la persona, con una perspectiva cronológica y biográfica (Kornblit 2004; Bertaux 1999), se tome a sí misma como objeto y se pueda mirar a distancia, formándose una conciencia reflexiva de lo vivido, deconstruyendo significados (Jiménez 2009) y reconstruyendo el sentido de las experiencias (Valdés y Gomariz 1992). Se trata de elementos de producción de sentido individuales que tienen una dimensión inmediatamente social (Díaz y Mella 2007; Nirenberg 2006; Petrlik 2008) y que son un reflejo de una época, de normas sociales y de valores compartidos con la comunidad de la que forma parte el sujeto (Pujadas 2000), como también de los cambios sociales (Bajtin 1999), históricos, económicos y políticos que influyen en su recorrido (Jiménez 2009).

Se realizaron 32 relatos de vida que examinaban la trayectoria laboral de los entrevistados desde el primer trabajo en Perú hasta el actual en Chile, indagando sobre las cualidades de cada uno, la decisión de migrar y la evaluación/expectativa personal de sus recorridos. El método para seleccionar a los entrevistados fue "bola de nieve", es decir, a partir de los contactos con los que se contaban, se buscaron nuevos informantes quienes permitieron identificar casos de interés. De esta manera, los criterios de selección fueron que inmigrantes peruanos tuvieran una trayectoria laboral ascendente y que hubiera igual cantidad de entrevistados hombres y mujeres, que actualmente estuvieran trabajando, tuvieran la experiencia de al menos dos trabajos en Chile y uno en Perú. ${ }^{6}$ El tiempo de migración, tipo de trabajo, edad, nivel educacional fueron variables que permitieron tener una muestra muy heterogénea.

\section{Los hitos críticos y las trayectorias laborales ascendentes}

La relevancia de analizar la trayectoria laboral como el movimiento de personas en el mercado del trabajo, se ve sustentada en la influencia de las estructuras sociales sobre la biografía de los sujetos según su clase, género, raza/etnia y nacionalidad, producto de procesos sociales y de las acciones desplegadas por los individuos (Bourdieu 1997). El resultado es una trayectoria laboral que implica cierta reconversión/reproducción de capital patrimonial (Jiménez 2009) y que, como proceso de incesantes cambios (Bour-

6 Las características de la muestra están detalladas en la tabla de anexo metodológico. 
dieu 1979), no asegura necesariamente continuidad ni ascendencia. La importancia de examinar los hitos críticos es que estos desmenuzan dicho trayecto como consecuencia de un conjunto de momentos clave que dan cuenta de la manera como se desenvuelven las personas en el mercado del trabajo (Correa 2004).

El análisis de la trayectoria laboral ascendente y los hitos críticos permite relevar la posición activa del sujeto en el trabajo, ya que incorpora no solo la forma como se delinean estrategias (Dávolos 2001) y la toma de decisiones personales en un espacio social determinado (Pries 1997), sino también expresa cómo los sujetos se apoyan en su capital social, cultural y económico (Bourdieu 1995), y combinan los antecedentes familiares, las relaciones personales y las condiciones del mercado de trabajo, para lograr movilidad laboral (Jiménez 2009). Los hitos críticos son elementos fundamentales de las trayectorias laborales que resumen procesos sociales e individuales y que dan cuenta de acciones, prácticas, posiciones y disposiciones en el trabajo (Valenzuela, Marquéz y Venegas 2001).

Estos puntos de inflexión son "sucesos que representan un cambio en la dirección del curso de la vida, en relación a la trayectoria pasada y que tienen un impacto en las probabilidades de los destinos de la vida futura" (Buontempo 2000). Situaciones clave tales como: la formación de pareja, el nacimiento de hijos (Guzmán, Arujo y Mauro 1999), la entrada al trabajo, la estabilidad de la participación, los cambios de empleo, las entradas, salidas, ascendencia y descendencia, son momentos que culminan en hitos que estructuran las trayectorias y los procesos de la vida de los sujetos (Godard 1996).

\section{Las diferencias de género en las trayectorias laborales}

Las trayectorias laborales iluminan los procesos de construcción y cambio en las relaciones entre hombres y mujeres al develar los distintos mecanismos de género que condicionan la experiencia en el trabajo y que afectan sus grados de libertad y autonomía para construir proyectos personales (Mauro 2004). Se analizarán las diferencias de género, no solo con el objetivo de conocer los hitos críticos en el recorrido laboral, sino también para comparar sus efectos en la ascendencia por separado, contextualizándolo al marco migratorio.

Las estructuras sociales de raza, clase social y género se intersectan en un sistema de estratificación dinámico que determina los privilegios que cada grupo obtiene (Mora 2008), vale decir, la estratificación social asigna significados específicos que en conjunto determinan un imaginario que hace plausible la oferta laboral determinada. La migra- 
ción de mujeres, entonces, obedece a motivaciones de diversa índole y además diferente a la de los hombres, desde aquellas estrictamente laborales, pasando por las de carácter familiar, hasta otras más individuales (Martínez 2003).

El género es una variable que organiza la migración (Pessar y Mahler 2001). Se arguye que, para las mujeres, las redes involucrarían decisiones migratorias cada vez más distanciadas del mero acompañamiento y seguimiento de sus parejas. De hecho, actualmente ha aumentado el número de mujeres que migran solas en busca de trabajo (Gabaccia 1992; Morokvásic 1984; Oliveira 2000). Se sugiere que más que una estrategia autónoma de surgimiento personal, la decisión de emigrar está vinculada a una estrategia de sobrevivencia, en especial debido a la condición de maternidad y al contexto familiar (Martínez 2003). Sin embargo, es necesario capturar la multifacética naturaleza de la migración y reconocer que las decisiones migratorias y la construcción social de la migración de las mujeres no son necesariamente las mismas que las de los hombres (Mora 2009).

\section{RESULTADOS}

De acuerdo a los relatos de vida analizados, se logró organizar la información según los puntos de inflexión que influyen en la trayectoria laboral. Dicha categorización fue útil para ordenar los hallazgos y dar cuenta de su riqueza, relacionando los hitos, los elementos de la ascendencia y la intensidad de cada dato para analizarlo en toda su dimensión.

Los cuatro tipos de hitos críticos relevantes en la trayectoria laboral ascendente de peruanos y peruanas entrevistados son denominados positivos en la medida en que posibilitan la ascendencia laboral, y negativos si limitan u obstaculizan la ascendencia en el trabajo, los cuales se conceptualizaron como: hitos laborales, hitos históricosociales, hitos familiares e hitos personales. Se analizó la incidencia de dichos puntos de inflexión en la ascendencia del recorrido laboral de inmigrantes, espacio donde se especifican los elementos clave que influyen en la culminación de hitos críticos en los itinerarios laborales, los cuales son: redes y tiempo de migración, contexto, capital simbólico, decisiones personales e intermitencia-linealidad de la trayectoria. Finalmente se especifican las similitudes y diferencias entre los puntos de inflexión que determinan la ascendencia de peruanos y peruanas.

Se encontraron tres elementos transversales a los relatos. En primer término, como característica de las trayectorias de los entrevistados se manifiesta que todas son 
intermitentes, es decir, implican varias salidas y entradas a distintos trabajos; también involucran altos y bajos en su recorrido. A pesar del tiempo de migración, el uso de redes, el capital simbólico o las características personales, los relatos de vida laboral expresan que las trayectorias en el trabajo cuentan con hitos críticos importantes que interrumpen su recorrido.

En segundo término, un factor que posibilita la ascendencia en las trayectorias es la utilización de redes en Perú y Chile; este se constituye como uno de los elementos más relevantes a la hora de ascender en el trabajo. Las redes no son solo un factor importante en la decisión de migrar, pues permiten establecer contacto con personas en Chile desde Perú, obtener información respecto de la situación laboral e incluso migrar con trabajo en Chile, sino también facilitan la entrada y estancia en el país de acogida, de manera tal que el inmigrante tiene mayor posibilidad de sociabilizar y establecer relaciones estratégicas para su inserción en el trabajo.

En último término, se develó otra característica relevante: las personas con recorridos ascendentes no se ubican en los espacios laborales que tradicionalmente se asocian a inmigrantes peruanos (Giorguli 2009; Texidó et al. 2003; Stefoni 2007; Ram 2008; Ruiz Tagle 1999; Cortés 2005; Mora 2008). Más bien, se encuentran situados en diferentes sectores dependiendo especialmente del rubro de su profesión, capital simbólico y contactos que poseen.

A continuación se definen y caracterizan los cuatro tipos de hitos críticos. Cada cual se describirá mediante ejemplos de patrones hallados en los relatos de vida laboral.

\section{Hitos laborales (externo)}

Procesos o momentos en el trabajo que marcan un punto de inflexión en la trayectoria laboral. Ejemplos de lo anterior son: ascendencia de un puesto a otro, entrada a un trabajo formal, primer contrato, aumentos de sueldo, estafas de socios y quiebra de empresas. Son hitos externos, pues son determinados por la decisión de otros.

Un ejemplo esclarecedor que contiene elementos clave de los hitos laborales es el de un peruano que lleva 16 años en Chile. Este relato da cuenta de importantes patrones hallados en las entrevistas: las trayectorias son un conjunto de momentos en la vida laboral con altos y bajos, e implican constantes entradas y salidas. Por otro lado, se manifiesta que los tipos de trabajos en los cuales se desenvuelven no siempre tienen relación con la profesión, ni tampoco con el trabajo anterior: 
Yo en Perú tuve mi fábrica de calzado para damas; después tuve que liquidarlo por circunstancia de los negocios. Aparte empecé a trabajar vendiendo libros, y después esa empresa se fusionó con la venta de cursos en inglés: trabajé más de 12 años ahí. Empecé como vendedor sin saber nada, incluso mis hermanos me decían "tanto que has estudiado", porque yo estudié administración de empresas, empecé como un simple auxiliar de contabilidad y al cabo de seis meses ya era gerente de ventas. (Hombre, 16 años en Chile, ingeniero)

Los hitos laborales son muy diversos y pueden marcar negativamente la trayectoria en el trabajo; sin embargo, los relatos de vida expresan que los inmigrantes logran revertir las situaciones negativas -haciendo uso de su capital simbólico y redes- convirtiéndolas en oportunidades para mejorar el escenario laboral en el país:

Así que fui a la imprenta y le dije: "Señor, mira, soy peruano, estoy indocumentado, no tengo casa, no tengo vehículo, crea en mí, lo único que tengo es mi fuerza de trabajo" y me dijo: "Voy a creer en ti, peruanito", y así empecé con la revista, trabajé durante 3 años, nos fue más o menos bien, después comencé a vender trabajos de imprenta, y actualmente tengo mi propia imprenta. (Hombre, 21 años en Chile, empresario)

Por otra parte, los hitos laborales y por ende las trayectorias, están marcados por la influencia del entorno familiar cercano. En el caso específico de las mujeres, sus trayectos también están fuertemente influidos por los recorridos de la pareja. Un ejemplo de lo anterior es el de una mujer que trabajaba en Perú, emigra a Chile por el trabajo del marido, luego debe salir de nuevo del mercado laboral chileno para cuidar a sus hijos y finalmente, con la ascendencia del marido (empresario), ella logra su propia ascendencia: ser administradora de un restaurante de él:

Aquí llegué, empecé de dueña de casa y tenía que salir a trabajar para poder apoyar a mi marido. Trabajé como año y medio. De ahí me retiré y me dediqué igual a mis hijos, y de ahí me puse a estudiar; estudié gastronomía cuatro años y medio, y actualmente estoy estudiando pastelería. Para poder apoyarme también aquí y mi marido, que tiene negocios, me apoya para poner este negocio y aquí trabajamos con mi hijo. (Mujer, 16 años en Chile, cocinera y empresaria)

Un hallazgo relevante es el uso de los grupos de interés como red laboral, es decir, la utilización de contactos que con el tiempo favorece la formación de una red. Por ejemplo, los restaurantes de comida peruana en Santiago, los dueños o administradores del rubro forman un grupo -al igual que los garzones o cocineros- quienes se relacionan, protegen e 
intercambian información. Lo anterior funciona como red en la medida en que les permite mejorar el negocio según sus intereses, lo que a la vez favorece su ascendencia:

Las personas que uno conoce por algo las conoce y hay que mantener la relación con las personas que he trabajado; se genera un tipo de relación de negocio, nunca los vas a perder[...] Tú haces las cosas porque quieres que te sirvan, porque quieres cierto reconocimiento. No todo en la vida es la plata; qué mejor que la gente que tú quieres, te reconozca el trabajo y se acuerde de ti por lo que hiciste, eso te da nuevas oportunidades. (Mujer, 10 años en Chile, chef encargada de un restaurante)

Otro elemento clave de los hitos laborales son las redes sociales establecidas con otros migrantes peruanos, facilitado por el tiempo de estancia en Chile; estas redes se han situado en distintos espacios laborales, lo que les ha permitido posicionarse en diversos grupos de inmigrantes peruanos en el país. De esta manera, logran conocer a personas que acceden a lugares similares, los espacios más visitados por peruanos y establecer lazos laborales importantes. Tal es el caso de peruanos que han formado grupos de compatriotas en Santiago, los cuales se reúnen periódicamente - como la Cofradía de Peruanos, las Damas Peruanas, la Hermandad del Señor de los Milagros, etc. Quienes también funcionan como referencia y red laboral para diversas necesidades.

\section{Hitos sociales (externo)}

Procesos o momentos de la historia social de Perú o Chile que marcan un punto de inflexión en la trayectoria laboral. Ejemplos de lo anterior son: crisis económica en Perú, amnistía en Chile, auge de la comida peruana en Chile.

Un hallazgo relevante es la regularización de la situación migratoria como requisito para la trayectoria ascendente. Se constituye como hito importante para los entrevistados la amnistía a peruanos y peruanas en Chile en el año 1998 y luego en 2008. Se entrevistaron dos casos que se vieron beneficiados por el proceso, lo cual marcó su llegada a Chile:

En todas las empresas he tenido contrato gracias a Dios. Pero igual me acogí a la Ley de Amnistía, se me hizo más fácil mi estadía aquí, se me hizo más fácil. Me acogí a la ley de amnistía del 2008. Entonces tener documentación era mucho más fácil. (Hombre, 12 años en Chile, técnico) 
Se expresa que la historia política en Perú ha determinado el funcionamiento económico del país y, por ende, también el mercado laboral hasta la actualidad:

Parece mentira, si pensamos hace 20 años nosotros tuvimos problemas graves en Perú, terrorismo, ahora esa revolución a la actualidad es una revolución de cocineros, revolución gastronómica, esa misma fuerza la verdad que se nota mucho en Perú. Hace 20 o 25 años más o menos atrás, era un drama, terrorismo, se tomó todo lo económico; ahora esto mismo está pasando en Perú, se cambia, los supermercados llenos de cocineros, buscando productos nuevos, frescos y de calidad, y eso nos va a potenciar para dar a conocer nuestra gastronomía al mundo. (Hombre, 10 años en Chile, chef internacional)

Los hitos en la historia de los países implicados producen efectos en los protagonistas, aun cuando son externos a sus decisiones personales, los entrevistados están insertos en un grupo social sin ser inmunes a procesos nacionales importantes. Por ende, cambios en la economía o de gobierno, políticas públicas y sociales e inclusive crisis mundiales, generan consecuencias en las trayectorias laborales; de ahí la relevancia del contexto social en los discursos individuales (Jiménez 2009; Bourdieu 1997; Dávolos 2001; Pries 1997). Por ejemplo, la “subversión” en Perú:

Yo hice la carrera de ingeniería eléctrica hasta el año 90, me tocó vivir la experiencia más dura, fue la etapa de la subversión, fue su pick en los años 90 con atentados y todo. Así que yo me despertaba en las mañanas con las explosiones. Después empecé a trabajar en Electro Perú, me vine a Chile, estudié un magíster en la Católica y actualmente, de manera paralela al trabajo, realizo investigaciones. (Hombre, 20 años en Chile, técnico eléctrico)

En toda situación laboral son determinantes las circunstancias en las cuales se desarrollan los trayectos. De acuerdo a los relatos analizados, el contexto influye directamente en la decisión de migrar y en el desempeño laboral de los trabajadores peruanos en Chile. Los procesos acontecidos en el país de origen y el receptor, pueden promover u obstaculizar las trayectorias en la medida en que establecen el marco normativo legal de los procesos políticos, económicos y sociales donde las personas evalúan sus oportunidades y expectativas.

Queda claro que el contexto de expansión económica y laboral en Chile abre posibilidades a inmigrantes peruanos y especialmente a aquellos que cuentan con recursos económicos, sociales y culturales, pues dichos capitales se valoran de manera creciente en 
el mercado laboral chileno, y así logran desenvolverse con menores dificultades. De esta manera, mejoran sus condiciones con respecto a Perú, contexto en el cual no hallaban posibilidades propicias para desarrollarse profesional, laboral o personalmente.

\section{Hitos familiares (interno)}

Procesos o momentos de familiares cercanos que marcan un punto de inflexión en la trayectoria laboral. Ejemplos de lo anterior son: separación de los padres, muerte de un familiar, emigración de la pareja por trabajo, nacimiento de hijos. Solo se tomaron en cuenta a los familiares que se encuentran en el entorno cercano al protagonista del relato, es decir, hijos, padres, parejas o familiares directos. Este tipo de hito es interno ya que influye en la vida, trayecto laboral y decisiones personales.

Un ejemplo de hito familiar es la separación de los padres de una inmigrante peruana, proceso que generó un conflicto en su entorno familiar cercano, lo que culminó en un hito crítico, es decir, su salida de Perú y llegada a Chile; lo anterior se transformó en una decisión favorable para su trayectoria, pues le permitió estudiar y ascender rápidamente. Este caso es además importante para demostrar la relevancia que en particular tienen las redes religiosas en Chile, contacto que a esta entrevistada le permitió entrar a estudiar:

Bueno, no pasó más que el primer semestre que estudié químico-farmacia y mi madre decidió traerme acá por problemas entre padres separados y yo, menor de edad, decidí venirme con mi madre. El primer semestre -yo llegué como en agosto- no se podía hacer nada acá, nada. Un hermano de la organización de los Testigos (de Jehová) me dio una posibilidad de poder entrar a estudiar. (Mujer, 10 años en Chile, técnico)

Lo anterior expresa la relevancia del origen familiar en conjunto con los recursos con los que cuentan los trabajadores antes de venir a Chile, sean económicos, sociales o culturales. La ascendencia está justamente dada por la utilización, actualización y acumulación de dichos recursos. En el caso de los entrevistados, los tres tipos de capital son por igual relevantes en las trayectorias. Los recursos en su conjunto son esenciales en ambos países y tienen un papel importante a la hora de conjugar las oportunidades de acuerdo a sus intereses en el trabajo, y su uso favorece ampliamente los recorridos y ascenso en el mercado laboral. 
Una situación que solo se pronuncia en los relatos de peruanas es que ellas cuentan con una amplia mayoría de hitos familiares en sus itinerarios laborales, lo que es desfavorable para su ascenso laboral:

Se quedaban mucho tiempo solos: los dos chiquitos estaban todo el día en el jardín y la otra lola salía igual en la tarde y tenía que apoyarla en el trabajo y de repente le mandaban tareítas en grupo. En ir a dejarla, comprar materiales que necesita y hasta eso igual, ver a los otros chiquitos, darles de comer y eso [...] Acá era muy difícil y para pagar a alguien, esa no era la idea tampoco. Si yo trabajaba era para apoyarlos más que todo. (Mujer, 16 años en Chile, cocinera y empresaria)

Lo particular de esta situación es que los hitos familiares marcan de tal manera los trayectos de las mujeres, que obstaculizan en gran medida su ascendencia pues se interrumpe por constantes salidas y entradas del trabajo, consecuencia de lo anterior es su inserción en trabajos más flexibles que no aseguran necesariamente su ascenso. Lo anterior impide la permanencia en el mismo trabajo o lograr construir un recorrido ascendente. Este es el caso de algunas mujeres peruanas que tienen problemas con el cuidado de sus hijos y con la inserción a ellos al jardín infantil en Chile (Stefoni 2007).

\section{Hitos personales (interno)}

Habilidades, actitudes y estrategias personales puestos en relación con las situaciones particulares de cada entrevistado y sus circunstancias, sumado a los condicionamientos externos son elementos que culminan en un hito personal. Ejemplos de lo anterior son: estudiar una carrera, realizar capacitaciones, entre otros.

Es decir, una característica individual en determinado contexto se manifiesta en habilidades, actitudes y estrategias que se ponen en juego en el ámbito laboral; resultado de lo anterior son hitos personales que posibilitan la ascendencia. Por ende, este tipo de hitos refleja la capacidad de un migrante de decidir asertivamente de acuerdo a su espectro de posibilidades que en una situación, circunstancia y momento particular, es posible de realizar en el trabajo. Es preciso aclarar que las decisiones personales no se toman aisladas de procesos familiares, sociales, históricos o estructurales; es decir, expresan situaciones externas donde es el protagonista del relato quien decide cuál es la dirección que tomará en determinado escenario.

La actitud hacia el trabajo se expresa, por ejemplo, en el cumplimiento de objetivos, colaboración con los compañeros de trabajo o resolución de problemas eficazmente; 
estas son cualidades que favorecen el desempeño laboral de inmigrantes quienes en su posición se ven obligados a demostrar mayor sumisión y obediencia. Se nota en los discursos un nivel de resignación y acostumbramiento a condiciones precarias de trabajo para su supervivencia, cualidades que deben presentar para habilitar sus capacidades en el trabajo (con el fin de mantener su contrato de trabajo en la mayoría de los casos, requerimiento para obtener su documentación definitiva).

En consecuencia, actitudes como la humildad, el esfuerzo, el trabajo arduo; aprendizajes como no caer en el juego de la discriminación, no hablar de los problemas históricos de Perú y Chile, y características personales son habilidades y conductas muy valoradas, lo cual culmina en puntos de inflexión para los recorridos laborales:

La humildad sobre todo, me gusta enseñar, me gusta aprender. Es un trabajo duro, donde se trabaja más de 7 horas y media diaria; no tenemos horario, nosotros trabajamos 15 a 18 horas diarias, tienes que tener una buena producción y una buena organización [...] eso se construye, no es de un día pa' otro tampoco. Hace algunos años fui al mercado, mirando una caja de pescados, y yo le dije: "Esto pa' mí no sirve, pa’ mí eso es basura". Entonces me dijo: "Peruanos conshetuma, qué te has creído". Entonces yo le digo: "Ya, gracias", y hasta ahí llegó. (Hombre, 10 años en Chile, empresario)

Otro hallazgo relevante corresponde al manejo de las situaciones como aprendizaje de la experiencia migratoria y laboral para mejorar las posibilidades de trabajo en Chile, de acuerdo al empleo en el que pretende desarrollarse:

Pero he aprendido también que cuando llego a un país tengo que aprender qué decir y qué no decir y eso es rico: cuando tú empiezas a encontrar el equilibrio en el punto de vista, cuando sabes manejar ambas cosas; por ejemplo, cuando yo estoy aquí en Chile y me preguntan, no sé po', traté de no ser muy pesada ni ser muy directa, ni hacer realce del país, ni tampoco achicar el país donde estoy viviendo. (Mujer, 10 años en Chile, técnico)

El modo en que los inmigrantes peruanos y peruanas utilizan sus oportunidades y opciones laborales, da cuenta de que durante su trayectoria aprenden a conjugar las posibilidades de la manera más conveniente posible, con el fin de trazar un recorrido en base a sus propósitos en Chile. La importancia de las actitudes personales en el contexto laboral está presente en todos los relatos de vida, donde los trabajadores peruanos aprenden en su recorrido a "comportarse" de acuerdo a los requerimientos del país de acogida, entendiendo cómo funciona la segregación laboral en Chile y especialmente la discriminación hacia el/la peruano/a. 
Se ha logrado captar que las trayectorias laborales no son lineales (Guzmán, Arujo y Mauro 1999), sino que son interrumpidas por hitos críticos -entradas y salidas, altos y bajos- influidos por diversos motivos -laborales, histórico-sociales, familiares o personales-, lo cual expresa que los recorridos ascendentes son heterogéneos, multicausados y con movilidad interna amplia; en ellos se imbrican procesos del contexto en el cual se desarrolla el trayecto y el capital con el que cuenta el protagonista. Los protagonistas hacen uso de sus experiencias para conjugar las posibilidades y oportunidades con sus expectativas, utilizando las redes y los contactos laborales, como también aprendiendo a manejar las situaciones adversas de tal modo que direccionan sus características personales, actitudes y habilidades positivamente en el mercado del trabajo, logrando como resultado una trayectoria ascendente.

\section{Las similitudes y diferencias entre peruanos y peruanas según los hitos críticos que determinan la ascendencia}

De acuerdo a la información obtenida en los relatos de vida laboral, se logró captar que en el caso de hombres y mujeres los hitos críticos adquieren importancia diferenciada y determinan la ascendencia laboral de diversas formas.

El capital cultural es determinante para la inserción laboral de hombres y mujeres en el mercado laboral chileno. Cursar algún tipo de carrera técnica, profesional o capacitación, ofrece a los trabajadores la oportunidad de optar a mejores tipos y/o condiciones de trabajo, lo que a la vez otorga más posibilidades de ascender laboralmente, pues se desarrollan habilidades, actitudes y conocimientos muy valorados en Chile.

En el caso de la sumatoria de los capitales -capital simbólico- hombres y mujeres cuentan con mayor posibilidad de acceder a nichos laborales más estables y con mejores condiciones. La familia de origen, el capital económico, las redes y contactos laborales, además de su nivel educacional, les permiten ocupar sus recursos favoreciendo su acceso al mercado laboral y promoviendo la capacidad de utilizar sus antecedentes previos para mejorar su trayecto futuro. Las redes son para todos los casos determinantes en la formación de un trayecto ascendente e influyen en la culminación de hitos críticos.

Sin embargo, avocándonos a las diferencias, se rescata el hecho de que los hombres entrevistados cuentan con mayor cantidad de hitos laborales. Lo anterior quiere decir que sus trayectorias están mayormente marcadas por procesos en el trabajo. Es necesario aclarar que la diferencia cuantitativa en los hitos laborales no implica necesariamente que los hombres trabajen más o menos o que el aspecto laboral se adjudique solo a ellos; 
más bien se alude a que las trayectorias de los hombres entrevistados están en su mayoría marcadas por hitos en el trabajo. En el caso de las mujeres entrevistadas, sus trayectorias están ampliamente determinadas por los hitos familiares y trayectorias de sus parejas.

En el caso de los hitos históricos y sociales, los entrevistados no son inmunes a procesos sociales importantes en Chile o Perú, por lo que no existen diferencias cuantitativas o cualitativas en este tipo de hitos. Se reafirma la relevancia del contexto en todos los relatos, pues influye sin exclusiones en la trayectoria laboral de inmigrantes peruanos en Chile. Esto confirma también la coherencia con el análisis de las trayectorias laborales, las cuales develan que los procesos sociales, históricos, estructurales, de género y familiares tienen un peso muy importante en la vida laboral de las personas.

Otra semejanza relativa a los procesos o momentos en la historia social de Perú o Chile que culmina en puntos de inflexión en los recorridos laborales es que, en ambos casos, influyen en mayor medida los procesos acontecidos en Perú que las situaciones difíciles en Chile, las que muchas veces determinan o influyen en la salida del país y la decisión de no volver por el momento.

Ahora bien, una similitud para hombres y mujeres de acuerdo a los hitos familiares, es que ambos se ven influidos por la familia de origen y entorno familiar. Sin embargo, en el caso de las mujeres entrevistadas, los hitos familiares marcan la trayectoria. Es necesario ser cuidadosos al inferir que estos hitos se asignarían mayormente a las mujeres, pues no podemos deducir de inmediato la reproducción de las nociones tradicionales de género. Este argumento refiere a una diferencia cualitativa y cuantitativa en hitos familiares que continúa con la noción de que la trayectoria laboral de las mujeres inmigrantes se ve marcada por eventos familiares -en Chile o Perú-, lo que no quiere decir que al hombre inmigrante no le influya en su vida personal.

Lo anterior indica que, de acuerdo a los relatos estudiados, las mujeres peruanas continúan ocupándose paralelamente de las preocupaciones laborales y familiares; esto se ve reflejado en la cantidad de hitos críticos. Consecuencia de lo anterior, en los casos que se entrevistaron, es que las mujeres salen de su trabajo para tener y cuidar a sus hijos, su hogar, familiares enfermos o porque el marido se lo pide, etc., hitos los hombres entrevistados. Lo anterior da cuenta del problema histórico para compatibilizar la vida familiar y laboral de las mujeres y, en consecuencia, su problema para ascender en su recorrido.

Sin embargo, los casos de trayectoria laboral ascendente son particulares. Las mujeres entrevistadas dan cuenta en su discurso de que sus labores familiares son igual de 
importantes que sus preocupaciones en el trabajo, por lo que es esencial para ellas no dejar de lado su carrera laboral e insertarse nuevamente cada vez que deben salir del mercado laboral. Cabe destacar que la mayoría de las mujeres que en la actualidad cuentan con capital simbólico elevado y que se ocupan además de su familia, poseen ayuda para realizar sus labores y cuidar hijos; en efecto cuentan con recursos para colocarlos en jardines o en colegios en los momentos en que están en horario laboral.

Otro elemento relevante que emerge de los relatos de vida laboral, es el caso -minoritario- de mujeres que no son casadas y/o no tienen hijos, producto de la dedicación exclusiva a sus carreras profesionales y laborales. Ejemplo de lo anterior son las mujeres jóvenes de 30 o 40 años solteras o separadas y sin hijos que argumentan no interesarse mayormente por una vida de casadas o con hijos, en algunos casos para tener más tiempo para dedicarse a ellas mismas y su trabajo, y en otros casos porque no es su mayor preocupación actualidad. Ambos casos son relevantes para el análisis, pues dan cuenta de situaciones en que las mujeres peruanas no necesariamente sacrifican a su familia por trabajo o su trabajo por la familia.

En los casos analizados, las trayectorias de las mujeres peruanas se ven marcadas por hitos familiares -y en el caso de los hombres por hitos laborales-; la ascendencia en la mayoría de los casos significa separación con la familia de origen o entorno cercano, es decir, la motivación de peruanos o peruanas de emigrar con este escenario, implica mejorar sus condiciones personales, sociales y económicas de vida con respecto a Perú.

Por otro lado, la ascendencia laboral en Chile para peruanos o peruanas, implica muchas veces trabajar 12 o 14 horas, fines de semanas o en lugares alejados de sus hogares, lo cual les resta tiempo en familia. Se arguye que para mujeres y hombres esto es significativo, ya que se vuelve poco compatible ser exitosos en su lugar de trabajo y dedicar paralelamente tiempo a sus familias. Lo anterior nos retrotrae al argumento tradicional de las dificultades de complementar ambas labores, especialmente para las mujeres; sin embargo, la particularidad es que en los casos de ascendencia laboral, la dificultad de conciliar el ámbito familiar con el laboral es mencionada tanto en hombres como en mujeres.

En el caso de peruanos y peruanas, sus trayectorias son intermitentes en la medida en que se ven interrumpidas por hitos en su mayoría laborales y familiares, respectivamente. Sin embargo, una diferencia que cabe destacar es que en el caso de los hitos laborales, en las trayectorias de hombres inmigrantes estas son mayoritariamente positivas y favorecen el recorrido y/o ascendencia, lo cual es diferente en el caso de las mujeres in- 
migrantes, para quienes sus trayectorias se ven interrumpidas por situaciones dilemáticas familiares, lo cual en la mayoría de los casos no favorece su ascendencia.

Los hitos personales son igualmente relevantes en la trayectoria para ambos, pues son características individuales las que les permiten conjugar sus aprendizajes en decisiones personales utilizando los recursos económicos, sociales y culturales, lo que conlleva a una trayectoria ascendente. La importancia de captar los discursos es que en ellos se vislumbran los condicionamientos internos y externos de la trayectoria laboral de inmigrantes en Chile, la cual refleja la forma como se conjugan de manera diferenciada los eventos históricos sociales y las situaciones particulares en discursos construidos subjetivamente.

\section{CONCLUSIONES}

La pregunta por los hitos críticos en las trayectorias ascendentes de inmigrantes peruanos en Chile logró iluminar un análisis respecto del comportamiento laboral de hombres y mujeres en el mercado laboral chileno, de acuerdo a los puntos de inflexión que intervienen en su ascenso.

En primer lugar, se presentó la inserción laboral de inmigrantes peruanos en Chile, fuertemente marcada por una migración laboral y femenina en Santiago que se inserta en servicios y comercio principalmente y que reproduce una segregación ocupacional influenciada por la discriminación de raza, clase y género, lo cual les otorga menos oportunidades de escalar en el mercado laboral. Por otra parte, se indagó sobre la nueva tendencia de inmigración peruana, caracterizada por profesionales, técnicos y personas de alta calificación que se insertan en sectores medios con mayor posibilidad de ascender.

En segundo lugar, se explicitó la pertinencia de analizar biográficamente el comportamiento laboral de los inmigrantes peruanos como resultado de elementos estructurales como también subjetivos, que se expresan como acumulación de experiencias, destrezas y conocimientos que van determinando en el tiempo diversas posiciones laborales sucesivas; no obstante, con incesantes transformaciones. En efecto, la perspectiva temporal de la trayectoria permite la concepción activa del sujeto que se moviliza de acuerdo a factores sociales, políticos, demográficos, de género y familiares, lo cual da forma y sentido al comportamiento laboral. El interés de investigar las trayectorias especialmente de acuerdo a los hitos críticos, es que estos resumen procesos históricos y sociales en sucesos relevantes que cambian la dirección de los recorridos laborales. 
En tercer lugar, se advirtió que las diferencias de género serían analizadas transversalmente como forma de estratificación social, pues organiza las experiencias en el marco migratorio y en el mercado laboral; de ahí que las estructuras sociales estén enraizadas por mecanismos de género que construyen proyectos laborales diferenciados.

Lo anterior, como se mostró, fue posible mediante el uso de una metodología cualitativa que busca captar los discursos y significaciones de hombres y mujeres en el mercado laboral. El método seleccionado fue biográfico, mediante la utilización de 32 relatos de vida laboral de 16 hombres y 16 mujeres.

Respecto de los resultados obtenidos, se concluirá de acuerdo a los tres objetivos planteados al comienzo, los cuales se responderán a continuación.

Los hitos críticos que condicionan la trayectoria laboral, de acuerdo a los discursos expresados en los relatos de vida, son: los hitos laborales, históricos y sociales, familiares y finalmente personales. Los hitos laborales (externos a las decisiones personales) son puntos de inflexión derivados de procesos en el trabajo. Estos inciden en la ascendencia, mientras más utilizan las redes y mientras aumenta el tiempo de migración, igualmente en el caso de hombres y mujeres. Ahora bien, tanto para hombres como para mujeres, existe un amplio capital cultural, el cual se ve reflejado en el tipo de trabajo. Sin embargo, una diferencia clara es que los hombres cuentan con una amplia mayoría de hitos laborales respecto de las mujeres, es decir, su trayectoria laboral está vigorosamente determinada por procesos en el trabajo. Otra diferencia es que las trayectorias de mujeres se ven fuertemente influidas por los hitos y recorridos laborales de sus parejas.

El segundo tipo son los denominados hitos históricos y sociales (externos a las decisiones personales), es decir, momentos clave en la historia social de Perú o Chile que culminan en puntos de inflexión en sus recorridos en el mercado laboral. La relevancia del contexto político, social y económico para las vidas laborales de las personas da cuenta de que las trayectorias individuales no son inmunes a dichos eventos nacionales. Ahora bien, no existen diferencias de género en la influencia en este tipo de hitos, aun cuando existe mayor incidencia de hitos críticos en Perú en comparación con los de Chile.

Los hitos familiares (determinados por decisiones personales) dan cuenta de procesos en la familia cercana que culminan en puntos de inflexión en los itinerarios laborales, los que están fuertemente ligados a la relevancia de los recursos económicos, sociales y culturales con los que cuentan los protagonistas. Ahora bien, respecto de las diferencias de género, las mujeres peruanas cuentan con mayor cantidad de hitos familiares, lo que 
incide en su menor ascenso, aun cuando existen algunos casos de mujeres sin expectativas de formar una familia en un mediano plazo. Finalmente, la ascendencia en todos los casos depende de la lejanía -momentánea o no- de la familia, ya sea en Perú o en Chile.

Por último, los hitos personales son habilidades, actitudes y estrategias personales puestos en relación con la situación, circunstancia y momento particular que se ponen en juego en el ámbito laboral y que culmina en un punto de inflexión de las trayectorias. En los discursos analizados, estas disposiciones están marcadas por un fuerte discurso de autoproducción del recorrido, donde se ponen en gran relieve las estrategias individuales por sobre las situacionales, percepción muy tradicional de trayectos con un difícil comienzo, situaciones dilemáticas y un ascenso claro.

Los hitos críticos revisados inciden directamente en el ascenso de peruanos y peruanas en Chile, en la medida en que hacen uso de las redes y contactos laborales, dependiendo de su elevado capital simbólico, lo cual está influenciado por el contexto en el cual toman sus decisiones y se desenvuelven en el país de llegada. Lo anterior genera como resultado trayectorias intermitentes pero ascendentes desde Perú hasta hoy. Lo anterior no asegura que las trayectorias estudiadas sean lineales o continúen igualmente ascendentes, pues el contexto o las situaciones dilemáticas imprevistas pueden provocar cambios en las posiciones sucesivas de las personas en el espacio laboral.

Los resultados de esta investigación son relevantes en la medida en que dan cuenta de la heterogeneidad de la inmigración laboral peruana a Chile, y también logran expresar el recorrido desde su primer trabajo en Perú hasta el actual. Por otra parte, se logra exponer la forma como se configuran las vidas laborales a través del tiempo. Finalmente, además de ofrecer una perspectiva diferenciada de hombres y mujeres, refleja momentos estructurales de la sociedad y etapas de las personas y sus significaciones, en eventos específicos o hitos críticos que, en su conjunto, conforman trayectorias laborales.

La pregunta por la ascendencia se aborda desde una perspectiva objetiva y subjetiva, lo que da cuenta de que la trayectoria laboral de los inmigrantes peruanos en Chile está determinada por los recursos (capitales) con que cuentan -teniendo en cuenta que su migración es económica y laboral. El ascenso está dado por la forma como las personas actualizan dichos recursos en la medida en que estos van siendo importantes según los eventos acaecidos durante su trayectoria. Por lo tanto, un hecho común se transforma en un hito cuando los protagonistas los significan de tal modo que aquellos les permiten sobrepasar una coyuntura específica, ya sea positiva o negativa, para luego resignificar su recorrido. 
Lo anterior explica por qué una trayectoria es ascendente para algunos casos y para otros no, y las razones por las cuales para hombres y mujeres estos puntos de inflexión influyen diversificadamente. Los hitos inciden en la trayectoria en la medida en que los recursos se acumulan en el tiempo de migración y logran ser activados para diversos usos de acuerdo a las circunstancias, puesto que los recursos económicos, sociales y culturales se reparten diferenciadamente en la sociedad -de acuerdo a la clase, raza/ etnia y género. Al final, la trayectoria laboral es favorable dentro del marco migratorio mientras aumenta y se acumulan los capitales; entonces, mayor y más certificada es la ascendencia laboral en Chile.

Sin embargo, cabe cuestionarse si este diagnóstico sería similar en el caso de hombres y mujeres chilenos desde una perspectiva de movilidad social, pues la segmentación ocupacional se repite en otros tipos de estratificación como la clase social y género. Aun cuando no existirían brechas por la nacionalidad, raza/etnia, efectivamente existe una inserción diferenciada en el trabajo que podría examinarse para una próxima investigación.

Finalmente, es pertinente plantear un cuestionamiento que surge luego de la presente investigación, y que tiene relación con la posible reconfiguración de las relaciones de género en el marco migratorio, en especial provocada por los cambios de posición que generan las trayectorias ascendentes. Lo anterior, dado que en los relatos analizados hombres y mujeres cambian sus discursos, percepciones y significaciones de su comportamiento laboral y también personal, lo que probablemente lograría reconfigurar la forma en que hombres y mujeres se relacionan en el ámbito personal y laboral.

\section{REFERENCIAS}

Araujo, Katya, Virginia Guzmán y Amalia Mauro. 2000. El surgimiento de la violencia doméstica como problema público y objeto de políticas. Santiago: CEM.

Bajtín, Mijaíl. 1999. Estética de la creación verbal. México D.F.: Siglo XXI.

Bertaux, Daniel. 1999. "El enfoque biográfico, su validez metodológica y sus potencialidades." Proposiciones 29:1-23

Bourdieu, Pierre. 1979. La distinction social sritique of the judgement of taste.. London: Routledge. 1984. La distinción. Madrid: Taurus. 
1985. La Reproducción. Barcelona: Laia.

1995. Respuestas por una antropología reflexiva. México D.F.: Grijalbo.

. 1997. Razones prácticas. Barcelona: Anagrama.

Bourdieu, Pierre y Jean- Claude Passeron. 1977. La reproducción: elementos para una teoría de la enseñanza. Barcelona: Editorial Laia.

Buontempo, María Paula. 2000. Inserción laboral de graduados universitarios: un estudio desde las trayectorias laborales. Corrientes: Universidad Nacional del Nordeste.

Coleman, James. 1998. Foundations of social theory. Massachusetts: Belknap Press.

Cortés, Patricia. 2005. Mujeres migrantes de América Latina y el Caribe: derechos humanos, mitos y duras realidades. Santiago: Programa Regional de Población y Desarrollo. Centro Latinoamericano y Caribeño de Demografía (CELADE).

Correa, Andrés. 2004: Trayectorias laborales y relacionales Una nueva estética. Cali: Grupo de Investigación en Evaluación y Calidad de la Educación Superior (GIECE), Universidad de San Buenaventura.

Crompton, Rosemary. 1994. Clase y estratificación. Una introducción a los debates actuales. Madrid: Tecnos.

Dávolos, Patricia. 2001. "Después de la privatización: trayectorias laborales de trabajadores con retiro voluntario." Revista Estudios del Trabajo 21:69-95.

Díaz, Estrella y Paola Mella. 2007. Inequidad y brechas de género en el empleo. Análisis de los resultados de la Encuesta Nacional (Quinta Versión). Santiago: División de Estudios, Dirección del Trabajo.

Erikson, Robert y John Goldthorpe. 1993. The constant flux: a study of class mobility in industrial societies. Oxford: Clarendon Press.

Gabaccia, Donna. 1992. Seeking common ground: multi-Disciplinary perspectives on immigrant women. Westport.: Greenwood Press.

Gioegulli, Silvia, Salvador Cobo y Francisco Alba. 2009. "La movilidad ocupacional de los migrantes de retorno: Un análisis comparativo entre países latinoamericanos.” En Migration 
in the Americas: Mexico and Latin America in comparative context, editado por Katharine Donato y Douglas Massey. Tenessee: Proyecto de Vanderbildt University.

Godard, Francis y Robert Cabanes. 1996. Uso de las historias de vida en las ciencias sociales. Colombia: Centro de Investigaciones sobre dinámica social, Universidad Externado de Colombia.

Goldthorpe, John. 1987. Social mobility and class structure in modern Britain. Oxford: Clarendon Press.

Guzmán, Virginia, Lthya Araujo y Amalia Mauro. 1999. Trayectorias laborales de mujeres: cambios generacionales en el mercado del trabajo. Santiago: CEM.

Henríquez, Helia y Verónica Riquelme. 2006. "Lejos del trabajo decente: el empleo desprotegido en Chile." Cuaderno de Investigación 30:1-180.

Jiménez, Mariela. 2009. “Tendencias y hallazgos en los estudios de trayectoria: una opción metodológica para clasificar el desarrollo laboral." Revista Electrónica de Investigación Educativa 11(1):1-21.

Kornblit, Ana Lía. 2004. "Historias y relatos de vida: una herramienta clave en metodologías cualitativas." Pp. 15-33 en Metodologías cualitativas en ciencias sociales, editado por Ana Kornblit. Buenos Aires: Editorial Biblos.

León, Arturo y Javier Martínez. 2001. La estratificación social chilena hacia fines del siglo XX. Santiago: CEPAL, Serie Políticas Sociales.

Martínez, Jorge. 2003. El mapa migratorio de América Latina y el Caribe, las mujeres y el género. Santiago: Proyecto Regional de Población CELADE UNFPA (Fondo de Población de las Naciones Unidas).

Mauro, Amalia. 2004. Trayectorias laborales en el sector financiero. Recorridos de las mujeres. Santiago: Unidad Mujer y Desarrollo, CEPAL.

Migueléz, Fausto. 2002. “PPor qué empeora el empleo?” Sistema: revista de ciencias sociales 168/169:37-52.

Mora, Claudia. 2008. “Globalización, género y migración.” Polis 7 (20):285-97.

. 2009. "Estratificación social y migración intrarregional: algunas caracterizaciones de la experiencia migratoria en Latinoamérica." Universum 24 (1):128-43. 
Morokvásic, Mirjana. 1984. "Birds of passage are also women." International Migration Review XVIII (4):886-907.

Nirenberg, Olga. 2006. "Los relatos de vida: herramientas útiles para la evaluación del impacto de la participación de adolescentes en proyectos." En Participación de adolescentes en proyectos sociales, editado por Nerinberg, Olga. Buenos Aires: Paidós.

Oliveira Assis, Gláucia de. 2000. "Rupturas e permanências: a emigração de brasileiros para os EUA e as transformaçoes nas relaçoes familiares e de gênero." XXIV Encontro Nacional da ANPOCS GT Familia e Sociedade, octubre. São Paulo Brasil.

Pessar, Patricia y Sarah Mahler. 2001. “Gender and transnational migration.” Artículo presentado en la conferencia Transnational Migration: Comparative Perspectives. Princeton, Princeton University.

Petrlik, Ana. 2008. Masculinidades en la tercera edad. Relatos de vida de varones adultos mayores que residen en un albergue de Lima. Lima: Universidad Nacional Mayor de San Marcos.

Pries, Ludger. 1997. "Conceptos de trabajo, mercados de trabajo y 'proyectos biográfico-laborales." Pp. 141-87 en Estudios sobre la cultura obrera en México, editado por De La O María Eugenia y Javier Melgoza. México: editorial Conaculta.

Pujadas, Juan J. 2000. "El método biográfico y los géneros de la memoria”. Revista de Antropología Social 9:127-58.

Ram, Monder. 2008. "Forms of capital, mixed embeddedness and Somali enterprise". Work, Employment \& Society September 22:427-46.

Servicio Nacional de la Mujer (Sernam). 2002. Conciliación entre vida laboral y vida familiar de trabajadores y trabajadoras chilenos/as. Santiago: Servicio Nacional de la Mujer, Departamento de Estudios y Estadísticas, Documento de Trabajo $N^{\circ} 76$.

. 2003. Análisis de los costos y beneficios de implementar medidas de conciliación de vida laboral y familiar en la empresa. Santiago: Servicio Nacional de la Mujer, Departamento de Estudios y Estadísticas, Escuela de Aministración de la Pontificia Universidad Católica de Chile, Documento de Trabajo $N^{\circ} 84$.

Stefoni, Carolina. 2007. "La migración en la agenda chileno-peruana. Un camino por construir." Pp. 551-64 en Nuestros vecinos, editado por Mario Artaza y Paz Milet. Santiago: RIL, Universidad de Chile. 
2009. "Inmigrantes en Chile. Una integración diferenciada al mercado laboral." Pp. 81-108, en Migración y políticas sociales en América Latina, editado por SOPLA, Konrad Adenauer Adenauer Stiftung. Rio de Janeiro: KAS.

Texidó, Ezequiel, et al. 2003. Migraciones laborales en Sudamérica: el Mercosur ampliado. Ginebra: OIT, Estudios sobre Migraciones Internacionales 63

Torche, Florencia y Guillermo Wormald. 2004. Estratificación y movilidad social en Chile: entre la adscripción y el logro. Santiago: CEPAL, Serie Políticas Sociales.

Valdés, Teresa y Enrique Gomariz, coord. 1994. Mujeres latinoamericanas en cifras. Santiago: Flacso Chile España, Ministerio de Asuntos Sociales- Instituto de la Mujer.

Valenzuela, M. Elena, Francisca Márquez y Silvia Venegas. 2001. "Construyendo microempresa en Chile: trayectorias laborales de hombres y mujeres." Revista Latinoamericana de Estudios del Trabajo 13 (7):139-59.

Wright, Erik. 1992. "Reflexionando, una vez más, sobre el concepto de estructura de clases." Zona Abierta 59-60:17-73. 


\section{Anexo metodológico: características de la muestra}

\begin{tabular}{|c|c|c|c|c|}
\hline Entrevistado & Edad & Lugar nacimiento & Años en Chile & Nivel educacional \\
\hline Hombre 1 & 26 & Lima & 2 & Secundaria completa \\
\hline Hombre 2 & 52 & Lima & 5 & Universitaria completa \\
\hline Hombre 3 & 39 & Paijan & 12 & Técnico completo \\
\hline Hombre 4 & 47 & Lima & 18 & Universitaria incompleta \\
\hline Hombre 5 & 40 & Lima & 10 & Técnico completo \\
\hline Hombre 6 & 61 & Lima & 20 & Universitaria completa \\
\hline Hombre 7 & 46 & Cosco & 17 & Universitaria completa \\
\hline Hombre 8 & 38 & Lima & 2 & Universitaria completa \\
\hline Hombre 9 & 40 & Lima & 15 & Secundaria completa \\
\hline Hombre 10 & 23 & Lima & 5 & Secundaria completa \\
\hline Hombre 11 & 54 & Cajamarca & 14 & Universitaria completa \\
\hline Hombre 12 & 26 & Lima & 2 & Técnico incompleto \\
\hline Hombre 13 & 54 & Lima & 8 & Primaria incompleta \\
\hline Hombre 14 & 56 & Huancayo & 4 & Primaria completa \\
\hline Hombre 15 & 33 & Lima & 9 & Secundaria completa \\
\hline Hombre 16 & 60 & Apurimac & 22 & Universitaria completa \\
\hline Mujer 1 & 21 & Lima & 2 & Secundaria completa \\
\hline Mujer 2 & 36 & Lima & 12 & Técnico completo \\
\hline Mujer 3 & 32 & Huancayo & 6 & Técnico completo \\
\hline Mujer 4 & 45 & Lima & 16 & Universitaria completa \\
\hline Mujer 5 & 39 & Lima & 10 & Universitaria completa \\
\hline Mujer 6 & 25 & Cajamarca & 16 & Técnico superior \\
\hline Mujer 7 & 50 & Lima & 16 & Universitaria incompleta \\
\hline Mujer 8 & 30 & Cajamarca & 5 & Universitaria completa \\
\hline Mujer 9 & 25 & Lima & 5 & Secundaria completa \\
\hline Mujer 10 & 28 & Lima & 2 & Universitaria completa \\
\hline Mujer 11 & 46 & Arequipa & 16 & Universitaria completa \\
\hline Mujer 12 & 33 & Lima & 5 & Universitaria completa \\
\hline Mujer 13 & 39 & Pisac & 2 & Primaria completa \\
\hline Mujer 14 & 49 & Lima & 15 & Secundaria completa \\
\hline Mujer 15 & 27 & Lima & 6 & Técnico incompleto \\
\hline Mujer 16 & 24 & Chimbote & 6 & Técnico completo \\
\hline
\end{tabular}

